\title{
Concomitant Acute Transverse Myelitis and Sensory Motor Axonal Polyneuropathy in Two Children: Two Case Reports
}

\author{
Hyung Chung, MD, Kyung-Lim Joa, MD, Hyo-Sang Kim, MD, \\ Chang-Hwan Kim, MD, Han-Young Jung, MD, Myeong Ok Kim, MD
}

Department of Physical and Rehabilitation Medicine, Inha University School of Medicine, Incheon, Korea

Acute transverse myelitis (ATM) is an upper motor neuron disease of the spinal cord, and concomitant association of peripheral polyneuropathy, particularly the axonal type, is rarely reported in children. Our cases presented with ATM complicated with axonal type polyneuropathy. Axonal type polyneuropathy may be caused by acute motorsensory axonal neuropathy (AMSAN) or critical illness polyneuropathy and myopathy (CIPNM). These cases emphasize the need for nerve and muscle biopsies to make the differential diagnosis between AMSAN and CIPNM in patients with ATM complicated with axonal polyneuropathy.

Keywords Acute transverse myelitis, Acute motor-sensory axonal polyneuropathy, Critical illness polyneuropathy, Critical illness myopathy

\section{INTRODUCTION}

Transverse myelitis is an acute or subacute disorder that induces segmental demyelination or necrosis at several spinal cord levels [1]. Patients with acute transverse myelitis (ATM) present with pain, fever, and a combination of motor, sensory, and sphincter dysfunctions thought to result from an autoimmune response, which may be idiopathic, or may be associated with post-infec-

Received May 13, 2014; Accepted July 29, 2014

Corresponding author: Myeong Ok Kim

Department of Physical and Rehabilitation Medicine, Inha University School of Medicine, 27 Inhang-ro, Jung-gu, Incheon 400-711, Korea

Tel: +82-32-890-2480, Fax: +82-32-890-2486, E-mail: rmkmo@inha.ac.kr

(c) This is an open-access article distributed under the terms of the Creative Commons Attribution Non-Commercial License (http://creativecommons. org/licenses/by-nc/3.0) which permits unrestricted noncommercial use, distribution, and reproduction in any medium, provided the original work is properly cited.

Copyright ( 2015 by Korean Academy of Rehabilitation Medicine tious or para-infectious phenomena [2,3]. ATM is generally an upper motor neuron disease of the spinal cord, but we experienced two pediatric cases with simultaneous and sequential axonal type polyneuropathy, which are lower motor neuron diseases. In these cases, acute motor-sensory axonal neuropathy (AMSAN) or critical illness polyneuropathy and myopathy (CIPNM) were suspected. We report two cases and emphasize the need for nerve and muscle biopsies to differentially diagnose AMSAN and CIPNM in patients with ATM complicated with axonal polyneuropathy.

\section{CASE REPORTS}

\section{Case 1}

A 10-year-old girl was admitted to the emergency department with acute lower extremity weakness. She had experienced a viral illness with fever 1 week earlier. 
Magnetic resonance imaging (MRI) of the brain and a viral antibody test were normal. The cerebrospinal fluid (CSF) white blood cell count was normal but CSF protein had increased (105 mg/dL; range, $12.0-60.0 \mathrm{mg} /$ $\mathrm{dL})$, and immunoglobulin $\mathrm{G}$ was elevated $(13.5 \mathrm{mg} / \mathrm{dL}$; range, $0.6-3.4 \mathrm{mg} / \mathrm{dL}$ ). A CSF gram stain, culture, viral polymerase chain reaction, and oligoclonal bands were negative. A high signal intensity lesion between $\mathrm{C} 2$ and the upper thoracic cord was seen on spinal MRI (Fig. 1), and she was diagnosed with ATM. The patient showed trace to poor grade in both upper extremities and poor grade in both lower extremities. Sensation was generally preserved in the upper and lower extremities. Lower limb reflexes were pathologically brisk. She presented with exacerbating muscle weakness following a 6-day course of intravenous high-dose methylprednisolone (1 g/day) and immunoglobulin $(0.4 \mathrm{~g} / \mathrm{kg} /$ day). She was admitted to the intensive care unit (ICU) and was placed on a ventilator on day 7 due to respiratory muscle weakness. Nerve conduction studies on admission day 30 showed loss of amplitude of compound muscle action potentials (CMAP), decreased or absent amplitude of sensory nerve action potentials (SNAP), and delayed conduction velocities (Table 1). Needle electromyography showed polyneuropathic changes in the limb muscles: acute denervation with positive sharp waves and fibrillation potentials in the resting state, and reduced recruitment and polyphasic motor unit potentials during the volitional state.

Her muscle strength improved 2 months after admission; manual muscle testing of the upper and lower extremities revealed a fair grade. However, she could not sit up for herself and had difficulty maintaining balance in a
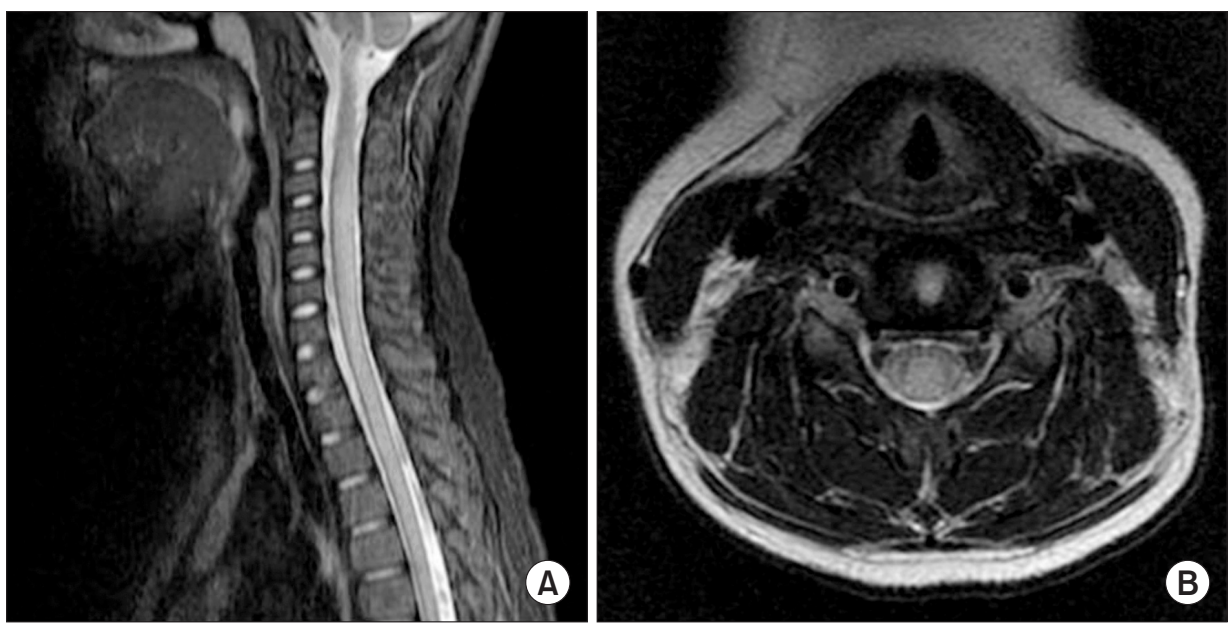

Fig. 1. Magnetic resonance images (MRI) of the cervical spine in case 1. (A) Sagittal section spinal MRI (post-gadolinium) reveals a high signal between $\mathrm{C} 2$ and the upper thoracic cord. (B) Transverse T2weighted MRI at the $\mathrm{C} 4$ cord level indicates cord edema.

Table 1. Nerve conduction studies of the two patients

\begin{tabular}{|c|c|c|c|c|c|c|}
\hline & \multicolumn{3}{|c|}{ Case 1} & \multicolumn{3}{|c|}{ Case 2} \\
\hline & Latency (ms) & Amplitude & Velocity $(\mathrm{m} / \mathrm{s})$ & Latency (ms) & Amplitude & Velocity $(\mathrm{m} / \mathrm{s})$ \\
\hline \multicolumn{7}{|l|}{ Motor nerve conduction study } \\
\hline Rt. Median nerve & $2.8 / 8.0$ & $8.6 / 7.9$ & $38.5^{\mathrm{a})}$ & $\mathrm{NE}^{\mathrm{a})}$ & $\mathrm{NE}^{\mathrm{a})}$ & $\mathrm{NE}^{\mathrm{a})}$ \\
\hline Rt. Ulnar nerve & $2.8 / 7.1$ & $9.4 / 8.0$ & $38.4^{\mathrm{a})}$ & $\mathrm{NE}^{\mathrm{a})}$ & $\mathrm{NE}^{\mathrm{a})}$ & $\mathrm{NE}^{\mathrm{a})}$ \\
\hline Rt. Deep peroneal nerve & $\mathrm{NE}^{\mathrm{a})}$ & $\mathrm{NE}^{\mathrm{a})}$ & $\mathrm{NE}^{\mathrm{a})}$ & $2.8 / 10.3$ & $2.6^{\mathrm{a})} / 2.3^{\mathrm{a})}$ & 41.3 \\
\hline Rt. Tibial nerve & $\mathrm{NE}^{\mathrm{a})}$ & $\mathrm{NE}^{\mathrm{a})}$ & $\mathrm{NE}^{\mathrm{a})}$ & $\mathrm{NE}^{\mathrm{a})}$ & $\mathrm{NE}^{\mathrm{a})}$ & $\mathrm{NE}^{\mathrm{a})}$ \\
\hline \multicolumn{7}{|l|}{ Sensory nerve conduction study } \\
\hline Rt. Median nerve & 2.4 & $2.9^{\mathrm{a})}$ & $38.4^{\mathrm{a})}$ & 3.0 & 24.0 & 40.0 \\
\hline Rt. Ulnar nerve & 2.9 & $4.0^{\mathrm{a})}$ & $39.7^{\mathrm{a})}$ & 2.9 & 27.0 & $39.1^{\mathrm{a})}$ \\
\hline Rt. Superficial peroneal nerve & $\mathrm{NE}^{\mathrm{a})}$ & $\mathrm{NE}^{\mathrm{a})}$ & $\mathrm{NE}^{\mathrm{a})}$ & $\mathrm{NE}^{\mathrm{a})}$ & $\mathrm{NE}^{\mathrm{a})}$ & $\mathrm{NE}^{\mathrm{a})}$ \\
\hline Rt. Sural nerve & $\mathrm{NE}^{\mathrm{a})}$ & $\mathrm{NE}^{\mathrm{a})}$ & $\mathrm{NE}^{\mathrm{a})}$ & $\mathrm{NE}^{\mathrm{a})}$ & $\mathrm{NE}^{\mathrm{a})}$ & $\mathrm{NE}^{\mathrm{a})}$ \\
\hline
\end{tabular}

Amplitudes are measured in millivolt ( $\mathrm{mV}$, motor) and in microvolt ( $\mu \mathrm{V}$, sensory).

NE, not evoked.

${ }^{\text {a) }}$ Represents abnormal data. 

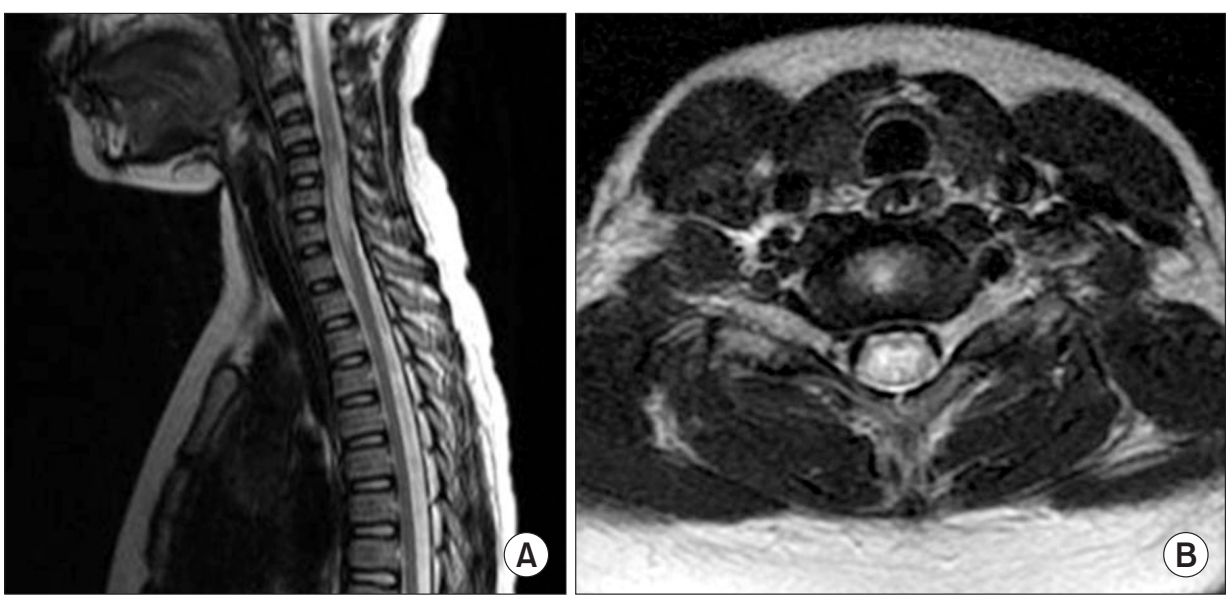

Fig. 2. Magnetic resonance images (MRI) of the cervical spine in case 2. (A) Sagittal section of spinal MRI (post-gadolinium) reveals a high signal between $\mathrm{C} 5$ and $\mathrm{C} 7$. (B) Transverse T2-weighted MRI at $\mathrm{C} 6$ indicates cord edema. sitting position. She could not expectorate sputum; thus, she was kept on a tracheostomy. The patient was suffering from ATM and concomitant involvement of axonal type polyneuropathy with a very poor prognosis.

\section{Case 2}

A previously well 10-year-old girl presented to the emergency department with acute general weakness and paresthesia. She had experienced a viral illness with fever 4 days earlier. Her muscle strength was fair to good in the upper extremities but nonexistent in all muscles of the lower extremities at admission. No sensory abnormalities were detected, but deep tendon reflexes had decreased in the upper and lower extremities. A high signal intensity lesion was observed at the C5-C7 cord level on sagittal and axial T2-weighted spine MRI (Fig. 2), and the diagnosis of ATM was made. After admission, the patient developed respiratory difficulty and a tracheostomy was done. The patient was administered highdose methylprednisolone ( $1 \mathrm{~g} /$ day) and immunoglobulin $(0.4 \mathrm{~g} / \mathrm{kg} /$ day) therapy for 10 days. Electrophysiological studies conducted on admission day 20 showed a loss or decreased amplitude of CMAP and a loss of amplitude of SNAP (Table 1). Needle electromyography revealed polyneuropathic changes in the limb muscles: acute denervation with positive sharp waves and fibrillation in the resting state, and reduced recruitment and polyphasic motor unit potentials during the volitional state. These electrophysiological abnormalities indicated axonal type polyneuropathy. She had gradual improvement in muscle strength over the next few weeks. A repeated manual muscle test 2 months after admission showed some improvement in hip flexion (fair), knee extension (fair), ankle dorsiflexion (poor), and ankle plantar flexion (poor). She showed concomitant ATM and involvement of polyneuropathy, which affected the axons more severely.

\section{DISCUSSION}

Transverse myelitis is characterized clinically by symptoms and signs of neurologic dysfunction in motor, sensory, and nerve tracts of the spinal cord. There may be a history of viral or febrile illness in the weeks preceding the onset of symptoms [2]. The complete pathophysiology of ATM is unknown, but it is generally believed to represent a demyelinating process [2].

AMSAN is a variant of Guillain-Barre syndrome (GBS), in which motor and sensory axons degenerate [3]. Simultaneous central and peripheral immune-mediated neural injury is rarely reported in case reports of combined ATM with AMSAN [4]. While most immune-mediated antibodies are directed at myelin proteins, axonal moieties may be the primary target of immune-mediated nerve injury in some cases [5]. CIPNM is an ICU-acquired weakness. CIPNM in a patient with ATM is another rarely encountered condition that includes motor and sensory abnormalities [6]. Because these two cases were treated in the ICU and suffered from neurophysiologic abnormalities due to motor and sensory axonal neuropathy, we considered AMSAN and CIPMN as peripheral causes of the neurophysiological abnormalities [7]. The differential diagnosis between CIPNM and AMSAN is difficult because of their similar clinical signs and symptoms and similar neurophysiologic abnormalities [7]. A differential diagnosis can be made based on the observation that weak- 
ness develops before admission to the ICU in patients with AMSAN [8]. However, concomitant ATM-induced limb weakness led to confusion in our cases, as we could not determine whether the weakness developed from CIPNM or AMSAN based on the clinical history. Because some evidence suggests that patients with ATM respond to steroids and those with GBS respond to immunoglobulin, combined treatment may be appropriate in those with possible or proven dual pathology [9]. However, corticosteroids are used before the onset of weakness in many CIPNM reports [10]. We cannot derive any causative association between these factors, as no controlled study was available. However, we could not exclude the possibility that corticosteroid use before admission to the ICU may have contributed to the CIPNM.

The AMSAN and CIPNM prognoses are not good. Many patients with AMSAN have a poorer prognosis than those with GBS, and they often cannot walk independently 6 months after onset [10]. In our patients, ATM with axonal type polyneuropathy could be an even worse prognosis. The prognosis for CIPNM is directly related to the prognosis for the underlying critical illness. Although the overall mortality rate is $36 \%-55 \%$, recovery can occur in patients who undergo intensive rehabilitation treatment to reduce the number of disabilities [10].

We report two pediatric cases diagnosed with ATM by spinal MRI and who showed axonal involvement of motor and sensory peripheral nerves on electrophysiologic studies. The neurophysiologic studies we conducted did not help us distinguish AMSAN from CIPNM. As nerve and muscle biopsies are infrequently performed in the ICU setting, the real cause of the axonal polyneuropathy remains undetermined.

Our cases presented with ATM complicated with axonal type polyneuropathy. Axonal type polyneuropathy may be caused by AMSAN or CIPNM. Nerve and muscle biopsies should be considered in patients in whom the CIPNM diagnosis is not entirely clear. Future studies are needed to evaluate efficient noninvasive diagnostic tools for CIPNM and to develop efficient medication for patients with ATM and CIPNM other than corticosteroids.

\section{CONFLICT OF INTEREST}

No potential conflict of interest relevant to this article was reported.

\section{ACKNOWLEDGMENTS}

This study was supported by an INHA University Research Grant.

\section{REFERENCES}

1. Berman M, Feldman S, Alter M, Zilber N, Kahana E. Acute transverse myelitis: incidence and etiologic considerations. Neurology 1981;31:966-71.

2. Kaplin AI, Krishnan C, Deshpande DM, Pardo CA, Kerr DA. Diagnosis and management of acute myelopathies. Neurologist 2005;11:2-18.

3. Griffin JW, Li CY, Ho TW, Xue P, Macko C, Gao CY, et al. Guillain-Barre syndrome in northern China: the spectrum of neuropathological changes in clinically defined cases. Brain 1995;118(Pt 3):577-95.

4. Howell KB, Wanigasinghe J, Leventer RJ, Ryan MM. Concomitant transverse myelitis and acute motor axonal neuropathy in an adolescent. Pediatr Neurol 2007;37:378-81.

5. Yuki N, Ang CW, Koga M, Jacobs BC, van Doorn $\mathrm{PA}$, Hirata $\mathrm{K}$, et al. Clinical features and response to treatment in Guillain-Barre syndrome associated with antibodies to GM1b ganglioside. Ann Neurol 2000;47:314-21.

6. Adamovic T, Willems A, Vanasse M, D'Anjou G, Robitaille Y, Litalien C, et al. Critical illness polyneuromyopathy in a child with severe demyelinating myelitis. J Child Neurol 2009;24:758-62.

7. de Letter MA, Visser LH, van der Meche FG, Ang W, Savelkoul HF. Distinctions between critical illness polyneuropathy and axonal Guillain-Barre syndrome. J Neurol Neurosurg Psychiatry 2000;68:397-8.

8. Yuki N, Hirata K. Relation between critical illness polyneuropathy and axonal Guillain-Barre syndrome. J Neurol Neurosurg Psychiatry 1999;67:128-9.

9. de Seze J, Lanctin C, Lebrun C, Malikova I, Papeix C, Wiertlewski S, et al. Idiopathic acute transverse myelitis: application of the recent diagnostic criteria. Neurology 2005;65:1950-3.

10. Visser LH. Critical illness polyneuropathy and myopathy: clinical features, risk factors and prognosis. Eur J Neurol 2006;13:1203-12. 